\title{
Breather-to-soliton conversions described by the quintic equation of the nonlinear Schrödinger hierarchy
}

\author{
A. Chowdury, D. J. Kedziora, A. Ankiewicz, and N. Akhmediev \\ Optical Sciences Group, Research School of Physics and Engineering, The Australian National University, Canberra, ACT 2600, Australia
}

(Received 5 November 2014; published 30 March 2015)

\begin{abstract}
We analyze the quintic integrable equation of the nonlinear Schrödinger hierarchy that includes fifth-order dispersion with matching higher-order nonlinear terms. We show that a breather solution of this equation can be converted into a nonpulsating soliton solution on a background. We calculate the locus of the eigenvalues on the complex plane which convert breathers into solitons. This transformation does not have an analog in the standard nonlinear Schrödinger equation. We also study the interaction between the new type of solitons, as well as between breathers and these solitons.
\end{abstract}

DOI: 10.1103/PhysRevE.91.032928

PACS number(s): 05.45.Yv, 42.65.Tg, 42.81.Qb

\section{INTRODUCTION}

Breathers introduce a new dimension into the seemingly well-known world of solutions of integrable equations. They have drawn much attention because of their interactions and energy exchange with a constant background [1-3]. Such interactions produce unique behaviors that are different from the usual soliton dynamics. Breathers are solutions that are periodic in space or in time and are classified as either Kuznetsov-Ma solitons [4] or Akhmediev breathers [5,6]. Each of these has been considered as a candidate for describing the notorious rogue waves [7]. The large-period limiting case of each is a Peregrine solution [8-10], which is localized both in space and time and which serves as a prototype of a rogue wave [11]. As such, it is known as a wave that "appears from nowhere and disappears without a trace" [12]. The Peregrine solution has been observed both in water waves [13] and in optics [14].

The nonlinear Schrödinger equation (NLSE) is a basic model for the propagation and dynamics of waves, both in optics $[15,16]$ and in water $[17,18]$. However, upon increasing the wave amplitude, we have to take into account higher-order effects which are not present in the basic model. These effects may add qualitatively new features to wave propagation phenomena [19]. Various higher-order terms have been considered [20-29]; they mostly result in nonintegrable equations which then lack analytic treatment. The latter are fundamentally important for understanding the wave propagation phenomena and for further progress in the area.

In this paper, we extend our studies to the quintic equation of the NLSE hierarchy. Preliminary analysis of this equation and its soliton solutions have been presented in Ref. [30]. Here, we present first-, second-, and third-order breather solutions of this equation, and the possibility of transforming them into continuous (nonperiodic) solitons on a background. The existence of such solutions is an unexpected feature of breathers. Various examples of collisions of these solitons and their interactions with ordinary breathers are given.

Painlevé analysis of modified NLS equations, which provides an indication of integrability, has been given in Ref. [31]. Preliminary analysis of this equation and its soliton solutions have been presented in Ref. [30]. The integrable NLSE with the addition of the third-order terms is known as the Hirota equation $[32,33]$. The equation with fourth-order terms is called the Lakshmanan-Porsezian-Daniel (LPD) equation [34-36]. The coefficients of higher-order terms of the LPD equation providing integrability were found by using the Painlevé analysis. This equation appears in the analysis of the Heisenberg spin chain. For an arbitrary combination of these operators of up to fourth order, soliton solutions were given in Ref. [37], while rogue waves were presented in Ref. [38].

The fifth-order equation of the NLSE hierarchy (QNLSH) including the third-order and fourth-order equation can be written in operator form as

$$
\begin{aligned}
& i \psi_{x}+S[\psi(x, t)]-i \alpha H[\psi(x, t)]+\gamma P[\psi(x, t)] \\
& \quad-i \delta Q[\psi(x, t)]=0,
\end{aligned}
$$

where the second-order term $S$ is the nonlinear Schrödinger operator,

$$
S[\psi(x, t)]=\frac{1}{2} \psi_{t t}+\psi|\psi|^{2},
$$

the third-order $H$ is the Hirota operator (beginning with thirdorder dispersion),

$$
H[\psi(x, t)]=\psi_{t t t}+6|\psi|^{2} \psi_{t},
$$

and the fourth-order $P$ is the Lakshmanan-Porsezian-Daniel (LPD) operator (beginning with fourth-order dispersion),

$$
\begin{aligned}
P[\psi(x, t)]= & \psi_{t t t t}+8|\psi|^{2} \psi_{t t}+6 \psi|\psi|^{4}+4 \psi\left|\psi_{t}\right|^{2} \\
& +6 \psi_{t}^{2} \psi^{*}+2 \psi^{2} \psi_{t t}^{*} .
\end{aligned}
$$

In the present work, we are mainly focusing on the fifthorder equation of the NLSE hierarchy. Taking only the quintic operator $[Q[\psi(x, t)]]$ from Eq. (1) with fifth-order dispersion, the equation of interest becomes

$$
i \psi_{x}+S[\psi(x, t)]-i \delta Q[\psi(x, t)]=0,
$$

and the fifth-order quintic operator can be given as

$$
\begin{aligned}
Q[\psi(x, t)]= & \psi_{t t t t t}+30|\psi|^{4} \psi_{t}+10|\psi|^{2} \psi_{t t t}+10 \psi \psi_{t} \psi_{t t}^{*} \\
& +10 \psi \psi_{t}^{*} \psi_{t t}+20 \psi^{*} \psi_{t} \psi_{t t}+10 \psi_{t}^{2} \psi_{t}^{*} .
\end{aligned}
$$

Here, $x$ is the propagation variable and $t$ is the transverse variable (time in a moving frame), with the function $|\psi(x, t)|$ being the envelope of the waves. We note that each term in Eq. (3) has an odd number of time derivatives. For example, the last term contains three first-order derivatives. This equation is part of the NLS hierarchy [39]. Selected solutions 
of this equation have been analyzed earlier in Ref. [40]. The Lax pair, conservation laws, and $N$-soliton solutions for a significantly restricted version of this equation, with real $\psi$, have been given in Ref. [41]. In this particular case, it is simply an extended Korteweg-de Vries equation, also applicable to surface waves in shallow water. In contrast, here we are dealing with complex $\psi$.

In optics, higher-order terms become progressively more important when we consider pulses of shorter duration propagating along a fiber. The general governing equation for pulse propagation in a fiber is given by [42]

$$
\begin{aligned}
i \psi_{x}= & -\sum_{m=1}^{\infty} \frac{i^{m} \beta_{m}}{m !} \frac{\partial^{m} \psi}{\partial t^{m}} \\
& -\gamma\left(1+i s \frac{\partial}{\partial t}\right)\left(\psi \int_{0}^{\infty} R\left(t^{\prime}\right)\left|\psi\left(t-t^{\prime}\right)\right|^{2} d t^{\prime}\right),
\end{aligned}
$$

where the first term on the right-hand side is the Taylor series expansion for linear dispersion, with corresponding coefficients $\beta_{m}$, while the second term describes the nonlinear terms, taking into account their dispersion. Here, $s$ is the self-steepening coefficient, the nonlinear term $\gamma$ depends on effective core area, and $R(t)$ includes instantaneous (electronic) and delayed nuclear (Raman) contributions of the nonlinear material response.

The integral in Eq. (4) is often approximated by taking the series to first order only, viz. $|\psi|^{2}-\tau_{R} \frac{\partial}{\partial t}\left(|\psi|^{2}\right)$, for Raman delay $\tau_{R}$. However, in reality, we need higher-order terms, and these involve higher-order time derivatives of intensity, $|\psi|^{2}$. In particular, $\psi \frac{\partial^{3}}{\partial t^{3}}\left(|\psi|^{2}\right)$ produces terms similar to those in $Q$. The coefficients for each term depend on the function $R\left(t^{\prime}\right)$ that, in turn, depends on the material parameters.

The quintic operator $Q$ contains all essential terms that may appear when we move beyond the basic NLSE approximation. The first term in Eq. (3) describes the linear fifth-order dispersion with $\delta=\beta_{5} / 120$. The other six terms describe the nonlinear dispersion. There is no clear way to associate any individual term in this equation with a single isolated physical phenomenon, as can be done for the third-order equation. Moreover, these terms can be rearranged in various forms. However, Eq. (3) contains the complete set of terms describing physical phenomena appropriate to that order. The relevance to a particular physical situation is also restricted by the choice of fixed coefficients in the operator. In reality, the coefficients may differ from those in Eq. (3). However, the coefficient $\delta$ in front of the whole operator can be varied and set close to an experimental value. This flexibility allows us to make reasonable adjustments for the actual physical phenomenon to be approximated in future experiments.

Importantly, the whole equation (1) is integrable and admits exact solutions. However, dealing with several free parameters at the very beginning is a cumbersome exercise. Therefore, we take a more cautious approach and first consider solutions with each operator separately before dealing with the whole equation, as it may produce excessively complicated solutions.

Considering the quintic term separately may be a reasonable approach, even from a practical point of view. As we know, modern optical fibers can be designed with various dispersion characteristics, and the lowest-order dispersion terms can be suppressed, making higher-order terms pivotal. Thus, the idea that certain predictions may allow experimental confirmations is not as outrageous as it may initially appear. Talking realistically, fifth-order dispersion does play a significant role in present laser experiments when pulses are close to $20 \mathrm{ps}$ in duration [43].

\section{BREATHER SOLUTION WITH COMPLEX EIGENVALUE}

The breather solutions of the fundamental NLSE have been analyzed in detail in Refs. [2,44-50]. The breather solution for the next-higher-order NLSE, which is well known as the Hirota equation, has been given in Ref. [3]. The fundamental property of this special solution, known as the Akhmediev breather, is that it is localized in " $x$ " and periodic in " $t$. " It describes the complete nonlinear evolution of an initial state that starts with modulation instability [48-50]. Solutions that have a small nonzero velocity have been dubbed "quasi-Akhmediev breathers" [47].

In the case of the NLSE, these solutions have a complex eigenvalue $\lambda$ of the inverse scattering transform theory, where the real part of the eigenvalue is responsible for the velocity, while the imaginary part characterizes the frequency of the modulation. Simple solutions like single-soliton or singlebreather solutions have one eigenvalue, while higher-order solutions may involve several eigenvalues. When using Darboux transformation schemes, each step adds one additional eigenvalue to the complex solution.

The periodicity plays an important role in the dynamics of the breather solution. The addition of higher-order odd terms to the NLSE results in oblique propagation of waves. Effectively, these terms change the velocity of the waves. The velocity change is proportional to the coefficient $\delta$ in the equation. Thus, a breather is subjected to reorientation in the $(x, t)$ plane. Below, we explicitly demonstrate how higher-order terms, specifically, the "quintic" operator, change the overall profile of breather solutions. The details of the mathematical calculations are given in Appendix.

To begin with, we present the first-order breather solution with complex eigenvalue $\lambda=a+i b$ :

$$
\psi_{1}=\left[1+2 b \frac{G_{1}+i H_{1}}{D}\right] e^{i x} .
$$

Here, the functions $G_{1}, H_{1}$, and $D$ are combinations of trigonometric and hyperbolic functions:

$$
\begin{aligned}
G_{1} & =\cos \left(x V_{T}+t \kappa_{r}\right) \cosh \left(2 \chi_{i}\right)-\cosh \left(x V_{H}+t \kappa_{i}\right) \sin \left(2 \chi_{r}\right), \\
H_{1} & =\cos \left(2 \chi_{r}\right) \sinh \left(x V_{H}+t \kappa_{i}\right)+\sin \left(x V_{T}+t \kappa_{r}\right) \sinh \left(2 \chi_{i}\right), \\
D & =\cosh \left(x V_{H}+t \kappa_{i}\right) \cosh \left(2 \chi_{i}\right)-\cos \left(x V_{T}+t \kappa_{r}\right) \sin \left(2 \chi_{r}\right),
\end{aligned}
$$

with

$$
\begin{aligned}
& \kappa=2 \sqrt{1+\lambda^{2}}=\kappa_{r}+i \kappa_{i} \\
& \chi=\frac{1}{2} \cos ^{-1}\left(\frac{\kappa}{2}\right)=\chi_{r}+i \chi_{i}
\end{aligned}
$$




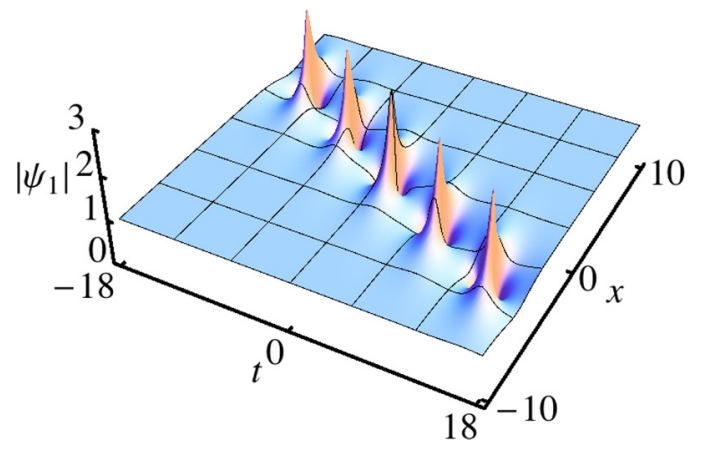

FIG. 1. (Color online) First-order quintic breather solution given by Eq. (5) with complex eigenvalue $\lambda=0.08+0.9 i$ and $\delta=\frac{1}{64}$.

and

$$
\begin{aligned}
\Omega & =16 a b\left(1-4 a^{2}+4 b^{2}\right), \\
\mho & =2\left[3+8 a^{4}+4 b^{2}+8 b^{4}-4 a^{2}\left(1+12 b^{2}\right)\right], \\
V_{T} & =-b \kappa_{i}+a \kappa_{r}+\delta\left(\Omega \kappa_{i}+\mho \kappa_{r}\right), \\
V_{H} & =a \kappa_{i}+b \kappa_{r}+\delta\left(\mho \kappa_{i}-\Omega \kappa_{r}\right) .
\end{aligned}
$$

This solution, for a fixed set of parameters, $\lambda=0.08+0.9 i$ and $\delta=\frac{1}{64}$, is illustrated in Fig. 1. As we will see in the next section, the actual value of the parameter $\delta$ does not change the effects that we observe qualitatively. It has to be sufficiently small in order to reflect the smallness of the higher-order terms in the QNLSH. Just as in the NLSE case, the imaginary part of the eigenvalue $\lambda$ is responsible for the period of the breather along the $t$ axis. This real part $a$ of the eigenvalue is equivalent to the velocity in the case of a soliton and thus induces a rotation of the total pulse train in the $(x, t)$ plane. The quintic terms controlled by the parameter $\delta$ introduce skewing of each individual peak profile in the breather. In the limiting case $\delta=0$, Eq. (2) reduces to the NLSE and the solution Eq. (5) reduces to the breather solution of the NLSE with a complex eigenvalue. As the equation we are dealing with is of fifth order with higher-order derivative terms in it, the solutions are extremely sensitive to the coefficient $\delta$. Therefore, we choose a fairly small $\delta$, e.g., $\frac{1}{16}$ or $\frac{1}{64}$, to generate visible effects in the solution.

\section{BREATHER-TO-SOLITON CONVERSION OF THE QNLSH SOLUTIONS}

In the NLSE case, Kuznetsov-Ma solitons $(b>1)$ and Akhmediev breathers $(0<b<1)$ can be transformed into each other by a change of the eigenvalue $\lambda$. Solutions stay periodic unless the period itself is taken to infinity; this occurs for $\lambda=i$, i.e., $a=0$ and $b=1$. In this case, each of these solutions is transformed into a Peregrine rational solution [51]. There are no solutions that describe continuous nonperiodic soliton propagation similar to standard solitons on a zero background. The situation changes when we add the quintic terms into equation. Then, specific values of the eigenvalue may transform a breather into a solution which is similar to an ordinary soliton solution. An example of such solution is shown in Fig. 2.

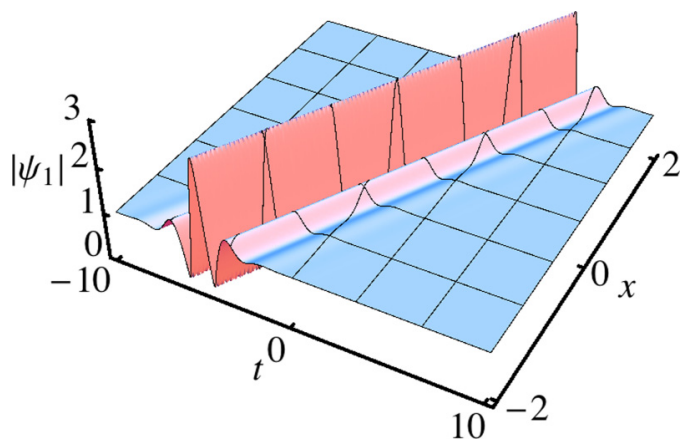

FIG. 2. (Color online) A breather transformed into a soliton. This solution is given by Eq. (5), with the eigenvalue $\lambda=-1.34329+0.9 i$. The propagation direction is $x$.

Below, we refer to all solitary waves which continuously propagate in $t$ as "solitons." In terms of collisions, they have the same properties as ordinary solitons of the NLSE. As will be shown in the following sections, they maintain the same velocity after a collision as they had before the collision. However, we should keep in mind that the solutions are on a constant background. The plane-wave background is modulationally unstable and a perturbation may distort the solutions formed on top of it. A complete study of the stability of such composite solutions is an issue that requires special investigation.

A transformation of a QNLSH breather, Eq. (5), into a continuous soliton occurs when we take

$$
\frac{V_{H}}{\kappa_{i}}=\frac{V_{T}}{\kappa_{r}} .
$$

In this case, the extrema of "cosine" and "cosh" functions in Eq. (5) are located along the same straight lines in the $(x, t)$ plane causing transformation of the breather into a continuous soliton.

Solutions of Eq. (6) can be represented on the complex plane $(a, b)$ for any particular $\delta$. After a simple calculation, we find that the condition of Eq. (6) can be reduced to a polynomial equation in $a$ and $b$ :

$$
16 a \delta\left(1-4 a^{2}+4 b^{2}\right)=1
$$

i.e., $\Omega \delta=b$. For an arbitrary real parameter $\delta$, Eq. (7) defines curves on the complex plane of $\lambda$, so that any point on these curves corresponds to a breather-to-soliton conversion. The easiest way to plot these curves is to take $a$ as the independent variable and find the solution for $b$ :

$$
b= \pm \sqrt{a^{2}-\frac{1}{4}+\frac{1}{64 a \delta}} .
$$

Curves calculated for positive $b$ for three different values of $\delta$ are presented in Fig. 3.

Alternatively, we can take $b$ as the independent variable and find the possible values of $a$ by solving a cubic algebraic equation Eq. (8). There are three branches of solution if $b$ is 


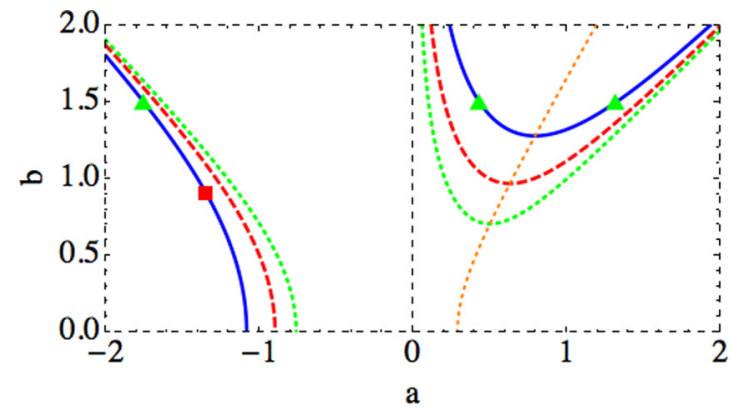

FIG. 3. (Color online) Solutions of Eq. (7) on a complex plane of $\lambda$ for (blue solid curves) $\delta=1 / 64$, (red long-dashed curves) $\delta=1 / 32$, and (green dotted curves) $\delta=1 / 16$. When $a>0$, part of a branch moves into the region of breathers $(b<1)$ when $\delta>$ $\frac{3}{80} \sqrt{\frac{3}{5}}=0.029 \cdots$. The large (red rectangle and green triangle) points on solution curves are the specific points where breathers become solitons. The (orange dotted) curve is the locus of the bifurcation points when $\delta$ changes [see Eq. (9)].

above the bifurcation point:

$$
\begin{aligned}
& a(1)=\frac{-8 \sqrt[3]{3}\left(4 b^{2}+1\right) \delta^{2}-\sqrt[3]{2} m^{2 / 3}}{4 \times 6^{2 / 3} \delta \sqrt[3]{m}}, \\
& a(2)=\frac{8(\sqrt{3}+3 i)\left(4 b^{2}+1\right) \delta^{2}+\sqrt[3]{2} \sqrt[6]{3}(1-i \sqrt{3}) m^{2 / 3}}{8 \times 2^{2 / 3} 3^{5 / 6} \delta \sqrt[3]{m}}, \\
& a(3)=\frac{8(\sqrt{3}-3 i)\left(4 b^{2}+1\right) \delta^{2}+\sqrt[3]{2} \sqrt[6]{3}(1+i \sqrt{3}) m^{2 / 3}}{8 \times 2^{2 / 3} 3^{5 / 6} \delta \sqrt[3]{m}},
\end{aligned}
$$

where $m=\delta^{2}\left[\sqrt{81-768\left(4 b^{2}+1\right)^{3} \delta^{2}}+9\right]$. Only one of these solutions, namely $a(1)$, is real if $b$ is below the bifurcation point.

At the bifurcation point, the value of $b$ is

$$
b_{0}=\frac{1}{2} \sqrt{\frac{3}{4(2 \delta)^{2 / 3}}-1} .
$$

This is the point where $d b / d a=0$ from Eq. (8), and also $a(2)=a(3)$. The value of $a$ at this point is

$$
a_{0}=\frac{1}{4(2 \delta)^{1 / 3}} \text {. }
$$

The curve giving the locus of bifurcation points, as $\delta$ changes, is

$$
3 a_{0}^{2}-b_{0}^{2}=\frac{1}{4}
$$

For the particular value of $\delta=1 / 64$, the bifurcation point is given by $a_{0}=0.793701$ and $b_{0}=1.28058$. This is the lowest point on the (blue) right-hand-side solid curve in Fig. 3.

To give an example, using the above solutions, we find that, for the same imaginary part as in Fig. 1, i.e., $b=0.9$, and for the same $\delta=1 / 64$, the real part of the eigenvalue is $a=-1.34329 \cdots$. This point is indicated by the lower solid-red rectangle on the left-most (blue) branch of the curves shown in Fig. 3. For this point $(\lambda)$ on the complex plane, a breather becomes a continuous soliton on a background. This soliton is shown in Fig. 2.

For a better understanding of this phenomenon, let us start with a simple case. When $a=0$, the velocity is zero for the

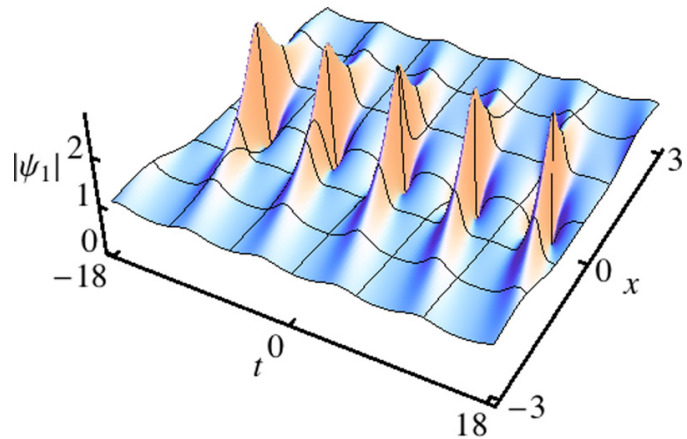

FIG. 4. (Color online) A breather given by Eq. (5) with the eigenvalue $\lambda=0+0.9 i$ and $\delta=1 / 16$. The propagation direction is $x$.

entire breather. The breather is parallel to the $t$ axis, while individual peak profiles can be skewed when $\delta$ is nonzero. An example is shown in Fig. 4. Here, in order to see more clearly the skewed position of each component, we have chosen a higher value, viz. $\delta=1 / 16$.

Deviations of " $a$ " from zero change the angle at which the breather as a whole is positioned relative to the $t$ axis. These deviations can be positive or negative. When this angle is equal to the angle of skewness of each peak, the breather becomes a soliton. For the parameters $b=0.9$ and $\delta=1 / 64$ this happens when $a=-1.34329 \cdots$. This is exactly the case shown in Fig. 2. Here, only one central peak of the periodic sequence remains. It becomes infinitely stretched along the direction of propagation. This stretched pulse appears as a soliton, as can be seen from Fig. 2. In this example, the soliton profile is symmetric. However, introducing translations which change the phase of the trigonometric functions may change the shape into an asymmetric or double-peak profile.

The cosine function provides oscillations of the envelope across the soliton, thus resulting in the oscillating tails in the orthogonal direction. Consequently, the soliton has tail oscillations in the form of a modulation of the background, as is clearly visible in Fig. 2. These oscillations form a distinctive feature of the solution, being different from the features of ordinary solitons of the NLSE on a zero background.

When $\delta \rightarrow 0$, Eqs. (6) and (7) do not have any solutions, showing that the NLSE does not allow conversion of breathers into new types of solitons. Only higher-order equations of the NLSE hierarchy admit such solutions. All of our examples are presented for the value of $\delta=1 / 64$ (except for the one in Fig. 4). This is a representative case that allows us to illustrate new solutions in the best way. Higher values of $\delta$ do not bring any new features in comparison to those shown in the plots given in this work.

\section{SECOND-ORDER BREATHER-TO-SOLITON CONVERSIONS}

If a first-order solution admits a breather-to-soliton transformation, we can expect similar transformations occurring in second-order solutions. Indeed, all the higher-order solutions are nonlinear superpositions of first-order solutions. Thus, if one or two components are transformed into solitons, we should obtain patterns similar to soliton interactions. 


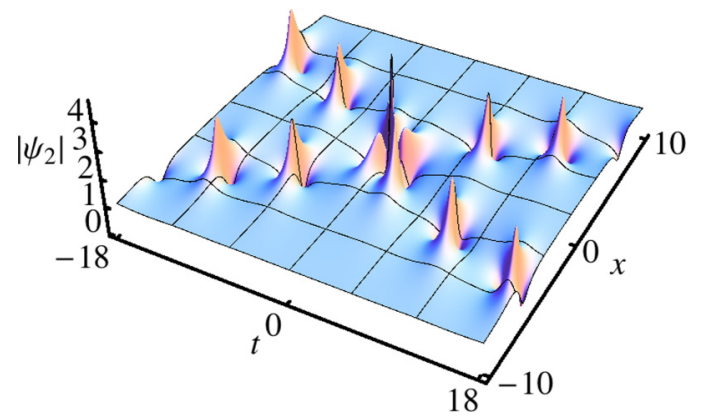

FIG. 5. (Color online) Two-breather solution with complex eigenvalues $\lambda_{1}=0.05+0.9 i$ and $\lambda_{2}=-0.05+0.9 i$, with $\delta=\frac{1}{64}$. Here, $x$ is the propagation direction.

For the second-order solution, we need two complex eigenvalues, $\lambda_{j}=a_{j}+i b_{j}$, where $j=1,2$. Details of the calculation are given in Appendix. We use the same representation of the Lax pair and the Darboux scheme for the QNLSH, Eq. (2), as in our previous work [30]. Figure 5 shows an example, with $\delta=1 / 64$, of a two-breather collision. In the particular case $\delta=0$, the solution reduces to the two-breather solution of the NLSE given previously in Ref. [2] by equation (22) of Appendix B. For comparison, the two-breather collision described by the NLSE can be found in Fig. 10 of Ref. [2].

In order to convert a two-breather solution into a two-soliton solution, we choose two arbitrary points $\lambda_{1}=\left(a_{1}, b_{1}\right)$ and $\lambda_{2}=$ $\left(a_{2}, b_{2}\right)$ on the curves in Fig. 3. In the following numerical example, we take as the first point $\lambda_{1}=-1.34329+0.9 i$, as already used in the previous section. Then, we take as the second point $\lambda_{2}$ with $b_{2}=0.8$ and $a_{2}=-1.2903$. We have chosen $\lambda_{2}$ different from $\lambda_{1}$. Equality would give a degenerate case which introduces additional complications which we do not want at the moment. An example of a breather collision converted to a soliton collision with these eigenvalues is shown in Fig. 6. This plot is indeed a two-soliton collision with the collision point located at the origin. The two solitons propagate in slightly different directions, due to the differences in $\lambda$.

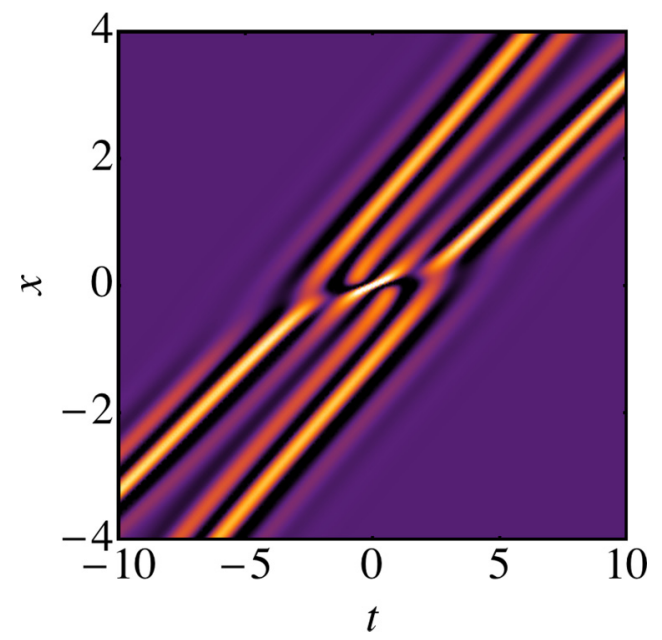

FIG. 6. (Color online) Contour plot of a second-order breatherto-soliton transformation. Here, the eigenvalues are $\lambda_{1}=-1.34329+$ $0.9 i$ and $\lambda_{2}=-1.2903+0.8 i$, while $\delta=\frac{1}{64}$.

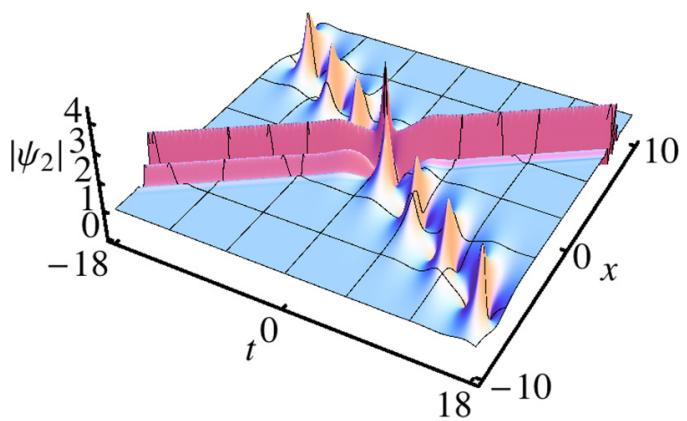

FIG. 7. (Color online) Collision between a breather with $\lambda_{2}=$ $0.2+0.8 i$ and a soliton with $\lambda_{1}=-1.34329+0.9 i$, when $\delta=\frac{1}{64}$.

Each soliton has an oscillating tail, thus creating a complicated collision pattern.

We also have the choice of converting only one of the components into a soliton, while allowing the other component to remain as a breather. Then we choose only one $\lambda$ located on a curve in Fig. 3, while the second $\lambda$ can be chosen arbitrarily on the complex plane. An example of a mixed collision of a breather and a soliton is shown in Fig. 7. The relative positions of the soliton and the breather can be controlled, not only by the eigenvalues, but also by translations of each component and changes in the phase of the breather. We omitted these from our calculations for simplicity. The highest amplitude, $\left|\psi_{2}\right|$, reached in the collision is $\left|\psi_{2}(0,0)\right|=4$.4. It is the same in both Figs. 6 and 7. In our examples, the maximum is located at the origin.

\section{HIGHER-ORDER SOLUTIONS}

Continuing the process of constructing higher-order breather-to-soliton converted solutions requires additional eigenvalues $\lambda_{j}$ located on any of the curves in Fig. 3. For example, in order to build a three-breather solution converted to a three-soliton solution, we have to choose three points on the curves in Fig. 3 or, equivalently, solve algebraic equations

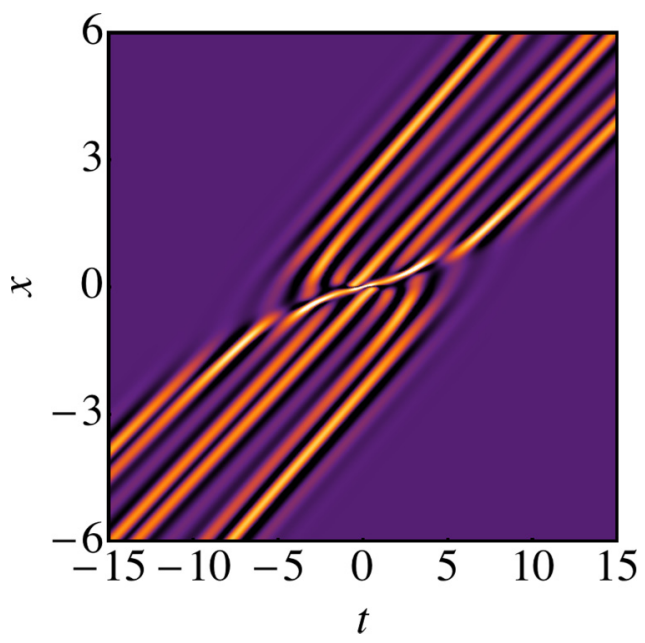

FIG. 8. (Color online) Contour plot of a third-order breather-tosoliton converted solution with three eigenvalues $\lambda_{1}=-1.3432+0.9 i$, $\lambda_{2}=-1.2903+0.8 i$, and $\lambda_{3}=-1.31616+0.85 i$, with $\delta=\frac{1}{64}$. 


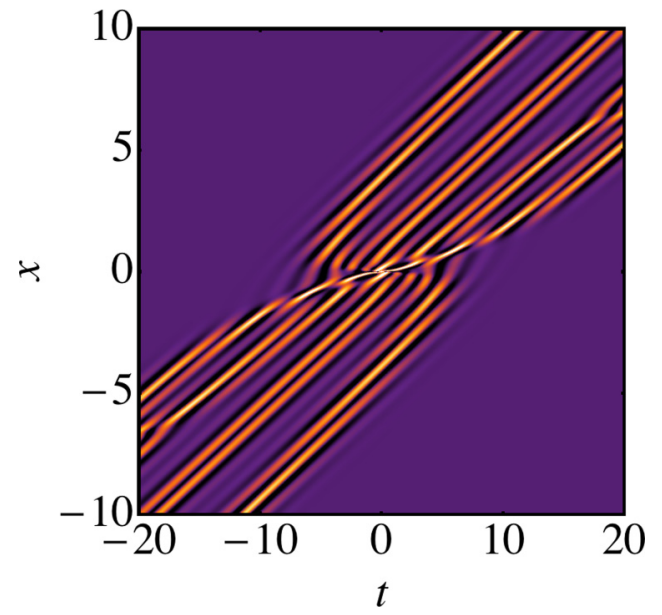

FIG. 9. (Color online) Contour plot of a fourth-order breather converted to four-soliton collision for $\lambda_{1}=-1.3432+0.9 i, \lambda_{2}=$ $-1.2903+0.8 i, \lambda_{3}=-1.3161+0.85 i$, and $\lambda_{4}=-1.30051+0.82 i$ with $\delta=\frac{1}{64}$.

(7) for three different eigenvalues. To be specific, if we have $a_{1}$ with $b_{1}$, and $a_{2}$ with a different $b_{2}$, we should generate one more $a_{3}$ with a different $b_{3}$. The choice of all three of these specific eigenvalues converts the three-breather solution into a three-soliton solution. Again, none of the eigenvalues can be identical if we want to avoid degenerate cases. The latter requires special consideration $[1,52]$ that is not presented here.

An example of a three-soliton collision built this way is shown in Fig. 8. This plot is constructed for three complex eigenvalues, $\lambda_{1}=-1.3432+0.9 i, \lambda_{2}=-1.2903+$ $0.8 i$, and $\lambda_{3}=-1.31616+0.85 i$. The highest amplitude at the point of collision here is $\psi_{3}(0,0)=6.1$. For a fourth-order soliton solution, we choose one more eigenvalue, $\lambda_{4}$ with $b_{4}=$ 0.82 and $a_{4}=-1.30051$, located on a curve in Fig. 3. Using the previous three eigenvalues, and this fourth eigenvalue, we can produce a fourth-order soliton solution from the four-order breather solution. It is presented in Fig. 9.

\section{KUZNETSOV-MA BREATHER CONVERSION TO SOLITON}

In the previous sections, we mainly presented the conversion of Akhmediev breathers to soliton solutions for the

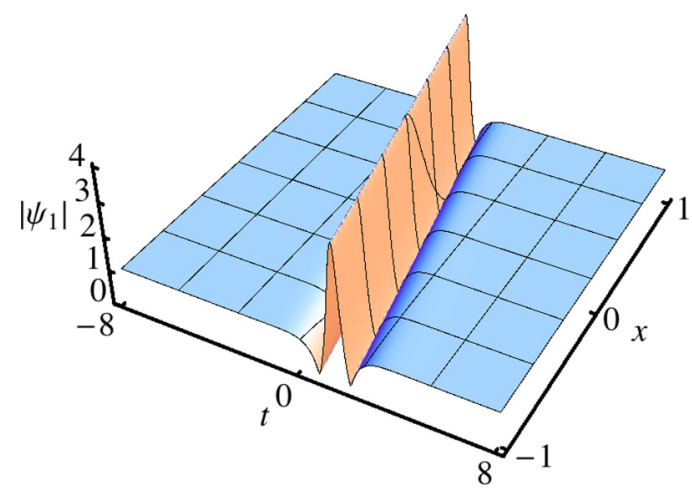

FIG. 10. (Color online) A Kuznetsov-Ma breather to soliton conversion for $\lambda=0.43232+1.5 i$ with $\delta=\frac{1}{64}$.

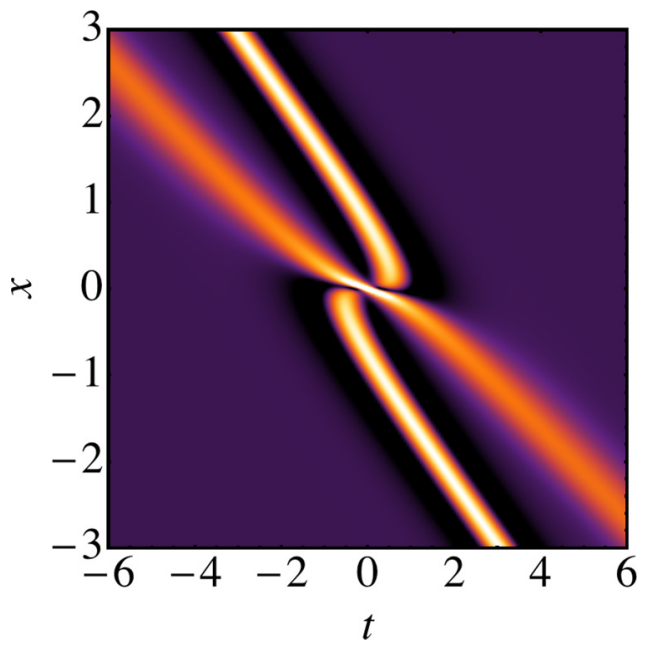

FIG. 11. (Color online) Collision of two solitons with eigenvalues $\lambda_{1}=0.43232+1.5 i$ and $\lambda_{2}=0.37457+1.6 i$, when $\delta=\frac{1}{64}$.

parameter range $0<b<1$. Apart from Akhmediev breathers, the conversion scenario also holds for Kuznetsov-Ma solitons when $b>1$. In this case, solving Eq. (7) leads to three real solutions for $a$. For given $b_{1,2,3}=1.5$, the solutions are $a_{1}=$ $-1.75233, a_{2}=0.43232$, and $a_{3}=1.32001$. These eigenvalues are shown in Fig. 3 by the large green triangle located at $b=1.5$ : one on the left branch (solid blue line) and the other two on the right branch (solid blue line). Each eigenvalue produces a continuous soliton. For example, the choice $a=a_{2}=$ 0.43232 produces the soliton which is shown in Fig. 10. This soliton has only one, infinitely stretched, peak. Two of these solitons propagate to the right while the one with $a=a_{2}=$ 0.43232 propagates to the left. Tail oscillations are damped for the latter case, and only one zero remains on each side of the soliton. This is clearly seen in Fig. 10. For lower values of $\delta$, the right-hand-side branch of the solutions in Fig. 3 moves down and three eigenvalues can exist, even for the case $b<1$.

Solitons of the right-hand-side branch can collide with each other or with solitons of the left-hand-side branch. An example

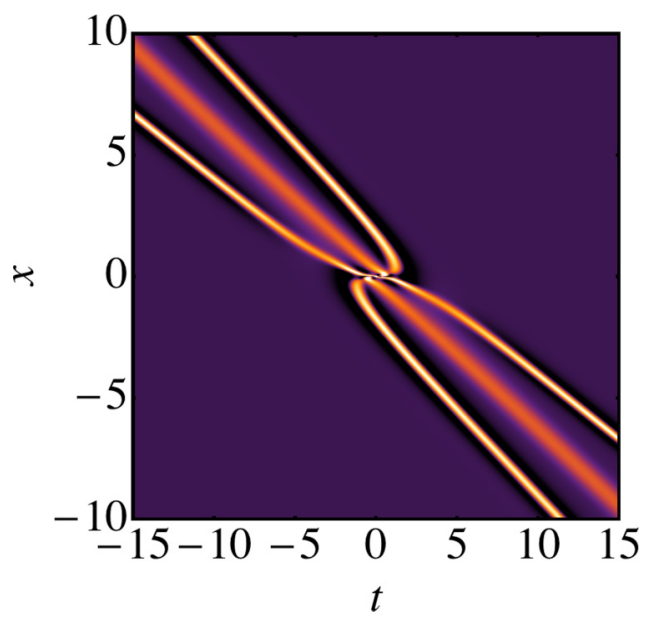

FIG. 12. (Color online) Three-soliton collision, where the eigenvalues are on a right-hand-side branch in Fig. $3: \lambda_{1}=0.43232+1.5 i$ and $\lambda_{2}=0.37457+1.6 i, \lambda_{3}=0.413193+1.53 i$. Here, $\delta=\frac{1}{64}$. 
of a collision of two solitons of the right-hand-side branch is shown in Fig. 11. Comparing this case with the plot in Fig. 8, we can notice that, first, the solitons propagate in a direction different from the one in Fig. 8. Second, the oscillatory tails are less visible. These features are in accordance with the properties of single solitons discussed above. The highest amplitude of the collision here is 7.2 at $(x, t) \rightarrow(0,0)$.

An example of a three-soliton collision is shown in Fig. 12. All eigenvalues here also belong to the right-hand-side branch of eigenvalues in Fig. 3.

\section{CONCLUSION}

We have derived first-order breather solutions of the QNLSH and have shown that they can be converted into solitons. The reasons and the conditions for conversion have been given explicitly. We calculated the locus of eigenvalues on the complex plane which convert the breathers into solitons. The solitons with these eigenvalues can collide as normal solitons on a zero background. We have provided several illustrations of such special solitons and patterns of their collisions.

\section{ACKNOWLEDGMENTS}

The authors acknowledge the support of the Australian Research Council (Discovery Project number DP140100265). N.A. and A.A. acknowledge support from the Volkswagen Stiftung. A.C. acknowledges Endeavour Postgraduate Award support.

\section{APPENDIX: DETAILS OF CALCULATIONS FOR THE FIRST- AND SECOND-ORDER BREATHER SOLUTIONS WITH COMPLEX EIGENVALUES}

Here, we follow our previous calculations, given explicitly in Appendices A and B of [2], except that the notation is adjusted to the present work. We separate the real and imaginary parts of $\kappa$ and $\chi$, so that $\kappa=\kappa_{j r}+i \kappa_{j i}$ and $\chi=\chi_{j r}+i \chi_{j i}$. We can express the linear $r$ and $s$ functions of Ref. [2] either in terms of exponential functions or trigonometric functions. To be specific, we will follow the latter case. Then $\lambda_{j}=a_{j}+i b_{j}, \kappa_{j}=2 \sqrt{1+\lambda_{j}^{2}}$, and $\chi_{j}=\cos ^{-1}\left(\frac{\kappa_{j}}{2}\right)$. We define $\kappa_{j r}=\operatorname{Re}\left(\kappa_{j}\right), \kappa_{j i}=\operatorname{Im}\left(\kappa_{j}\right), \chi_{j r}=\operatorname{Re}\left(\chi_{j}\right), \chi_{j i}=$ $\operatorname{Im}\left(\chi_{j}\right)$. Then linear functions $r$ and $s$ are

$$
\begin{aligned}
& r_{1,1}=2 i e^{-i x / 2} \sin (G), \\
& s_{1,1}=2 e^{i x / 2} \cos (H) .
\end{aligned}
$$

We can split the argument of the trigonometric functions as $G=A_{r}+i A_{i}$ and $H=B_{r}+i B_{i}$, where the real and imaginary parts are

$$
\begin{aligned}
& A_{r}=\chi_{1 r}+\frac{1}{2}\left(\kappa_{1 r} t+d_{1 r} x\right)-\frac{\pi}{4}, \\
& A_{i}=\chi_{1 i}+\frac{1}{2}\left(\kappa_{1 i} t+d_{1 i} x\right), \\
& B_{r}=-\chi_{1 r}+\frac{1}{2}\left(\kappa_{1 r} t+d_{1 r} x\right)-\frac{\pi}{4}, \\
& B_{i}=-\chi_{1 i}+\frac{1}{2}\left(\kappa_{1 i} t+d_{1 i} x\right) .
\end{aligned}
$$

Here $d_{1}=d_{1 r}+i d_{1 i}$ is the coefficient of $x$ in the argument.
Unlike the case of the NLSE, for the quintic equation $d_{1 r}$ and $d_{1 i}$ can be written in the form

$$
\begin{aligned}
& \Omega_{1}=16 a_{1} b_{1}\left(1-4 a_{1}^{2}+4 b_{1}^{2}\right), \\
& \mho_{1}=2\left[3+8 a_{1}^{4}+4 b_{1}^{2}+8 b_{1}^{4}-4 a_{1}^{2}\left(1+12 b_{1}^{2}\right)\right], \\
& d_{1 r}=-b_{1} \kappa_{1 i}+a_{1} \kappa_{1 r}+\delta\left(\Omega_{1} \kappa_{1 i}+\mho_{1} \kappa_{1 r}\right), \\
& d_{1 i}=a_{1} \kappa_{1 i}+b_{1} \kappa_{1 r}+\delta\left(\mho_{1} \kappa_{1 i}-\Omega_{1} \kappa_{1 r}\right) .
\end{aligned}
$$

Thus we obtain the solution

$$
\psi=e^{i x}\left[1+\frac{8}{D_{1}} i b_{1} \cosh \left(B_{i}-i B_{r}\right) \sinh \left(A_{i}+i A_{r}\right)\right],
$$

where $D_{1}=\cos \left(2 B_{r}\right)+\cosh \left(2 A_{i}\right)+\cosh \left(2 B_{i}\right)-\cos \left(2 A_{r}\right)$. The form of this solution looks similar to Eq. (18) of Appendix A in Ref. [2]. However, the arguments of the functions are quite different.

Similarly, for the second-order solution, the linear functions $r_{1,2}$ and $s_{1,2}$ are $r_{1,2}=2 i e^{-i x / 2} \sin (C), s_{1,2}=2 e^{i x / 2} \cos (D)$, where

$$
\begin{aligned}
& d_{2}=d_{2 r}+i d_{2 i}, \\
& C_{r}=\chi_{2 r}+\frac{1}{2}\left(\kappa_{2 r} t+d_{2 r} x\right)-\frac{\pi}{4}, \\
& C_{i}=\chi_{2 i}+\frac{1}{2}\left(\kappa_{2 i} t+d_{2 i} x\right), \\
& D_{r}=-\chi_{2 r}+\frac{1}{2}\left(\kappa_{2 r} t+d_{2 r} x\right)-\frac{\pi}{4}, \\
& D_{i}=-\chi_{2 i}+\frac{1}{2}\left(\kappa_{2 i} t+d_{2 i} x\right) .
\end{aligned}
$$

Here, $d_{2}$ is the coefficient of $x$ in the argument of the cos and $\sin$ functions in $r_{1,2}$ and $s_{1,2}$, while the arguments are separated into real and imaginary parts: $C=C_{r}+i C_{i}$ and $D=D_{r}+i D_{i}$.

Now, in order to derive the second-order quintic breather solution, we finally need to derive $r_{2,1}$ and $s_{2,1}$ in terms of $r_{1,1}$, $s_{1,1}, r_{1,2}$, and $s_{1,2}$. The coefficient of $x$, which is $d_{2}$, will have the same set of variables $\Omega_{2}$ and $\mho_{2}$ in its real and imaginary parts $\left(d_{2 i}, d_{2 r}\right)$, as in the first-order solution:

$$
\begin{aligned}
& \Omega_{2}=16 a_{2} b_{2}\left(1-4 a_{2}^{2}+4 b_{2}^{2}\right), \\
& \mho_{2}=2\left[3+8 a_{2}^{4}+4 b_{2}^{2}+8 b_{2}^{4}-4 a_{2}^{2}\left(1+12 b_{2}^{2}\right)\right], \\
& d_{2 r}=-b_{2} \kappa_{2 i}+a_{2} \kappa_{2 r}+\delta\left(\Omega_{2} \kappa_{2 i}+\mho_{2} \kappa_{2 r}\right), \\
& d_{2 i}=a_{2} \kappa_{2 i}+b_{2} \kappa_{2 r}+\delta\left(\mho_{2} \kappa_{2 i}-\Omega_{2} \kappa_{2 r}\right) .
\end{aligned}
$$

On taking these variables into account, the new linear functions $r_{2,1}^{*}$ and $s_{2,1}$ are

$$
\begin{aligned}
r_{2,1}^{*}= & -\frac{2}{D_{1}} e^{i x / 2}\left[4 i b_{1} \cosh \left(B_{i}-i B_{r}\right) \cosh \left(D_{i}+i D_{r}\right)\right. \\
& \times \sinh \left(A_{i}+i A_{r}\right)+\sinh \left(C_{i}+i C_{r}\right) \\
& \times\left\{\cosh \left(2 A_{i}\right)\left[-a_{1}+a_{2}+i\left(b_{1}-b_{2}\right)\right]\right. \\
& +\cos \left(2 A_{r}\right)\left[a_{1}-a_{2}-i\left(b_{1}-b_{2}\right)\right] \\
& \left.\left.+\left[\cos \left(2 B_{r}\right)+\cosh \left(2 B_{i}\right)\right]\left[-a_{1}+a_{2}-i\left(b_{1}+b_{2}\right)\right]\right\}\right],
\end{aligned}
$$




$$
\begin{aligned}
s_{2,1}= & -\frac{2}{D_{1}} e^{i x / 2}\left[4 i b_{1} \cosh \left(B_{i}-i B_{r}\right) \sinh \left(A_{i}+i A_{r}\right) \sinh \left(C_{i}-i C_{r}\right)+\cosh \left(D_{i}-i D_{r}\right)\left\{\cosh \left(2 A_{i}\right)\left[a_{1}-a_{2}-i\left(b_{1}+b_{2}\right)\right]\right.\right. \\
& \left.\left.+\cos \left(2 A_{r}\right)\left[-a_{1}+a_{2}+i\left(b_{1}+b_{2}\right)\right]+\left[\cos \left(2 B_{r}\right)+\cosh \left(2 B_{i}\right)\right]\left[a_{1}-a_{2}+i\left(b_{1}-b_{2}\right)\right]\right\}\right] .
\end{aligned}
$$

By using a recurrence relation for higher-order solutions in the Darboux scheme, we can derive the second-order quintic breather solution, including the real part of the eigenvalue. With $\delta=0$, this solution is a direct analog of the solution given in Appendix B, Eq. (22) of Ref. [2].

[1] N. Akhmediev and A. Ankiewicz, Solitons, Nonlinear Pulses and Beams (Chapman and Hall, London, 1997).

[2] N. Akhmediev, J. M. Soto-Crespo, and A. Ankiewicz, Extreme waves that appear from nowhere: on the nature of rogue waves, Phys. Lett. A 373, 2137 (2009).

[3] Yongsheng Tao and Jingsong He, Multisolitons, Breathers, and rogue waves for the Hirota equation generated by the Darboux transformation, Phys. Rev. E 85, 026601 (2012).

[4] B. Kibler, J. Fatome, C. Finot, G. Millot, G. Genty, B. Wetzel, N. Akhmediev, F. Dias, and J. M. Dudley, Observation of Kuznetsov-Ma soliton dynamics in optical fibre, Sci. Rep. 2, 463 (2012).

[5] J. M. Dudley, F. Dias, M. Erkintalo, and G. Genty, Instabilities, breathers and rogue waves in optics, Nat. Photon. 8, 755 (2014).

[6] A. Chabchoub, B. Kibler, J. M. Dudley, and N. Akhmediev, Hydrodynamics of periodic breathers, Philos. Trans. R. Soc., A 372, 20140005 (2014).

[7] K. B. Dysthe and K. Trulsen, Note on breather type solutions of the NLS as models for freak-waves, Phys. Scr. 1999, 48 (1999).

[8] D. H. Peregrine, Water waves, nonlinear Schrödinger equations and their solutions, J. Austral. Math. Soc. Ser. B 25, 16 (1983).

[9] K. L. Henderson, D. H. Peregrine, and J. W. Dold, Unsteady water wave modulations: fully nonlinear solutions and comparison with the nonlinear Schrödinger equation, Wave Motion 29, 341 (1999).

[10] C. Kharif, E. Pelinovsky, and A. Slunyaev, Rogue Waves in the Ocean (Springer-Verlag, Berlin-Heidelberg, 2009).

[11] V. I. Shrira and V. V. Geogjaev, What makes the Peregrine soliton so special as a prototype of freak waves? J. Eng. Math. 67, 11 (2010).

[12] N. Akhmediev, A. Ankiewicz, and M. Taki, Waves that appear from nowhere and disappear without a trace, Phys. Lett. A 373, 675 (2009).

[13] A. Chabchoub, N. P. Hoffmann, and N. Akhmediev, Rogue wave observation in a water wave tank, Phys. Rev. Lett. 106, 204502 (2011).

[14] B. Kibler, J. Fatome, C. Finot, G. Millot, F. Dias, G. Genty, N. Akhmediev, and J. M. Dudley, The Peregrine soliton in nonlinear fibre optics, Nat. Phys. 6, 790 (2010).

[15] A. Hasegawa and F. Tappert, Transmission of stationary nonlinear optical pulses in dispersive dielectric fibers, Appl. Phys. Lett. 23, 142 (1973).

[16] A. Hasegawa and Y. Kodama, Solitons in Optical Communications (Oxford University Press, New York, 1995).

[17] D. J. Benney and A. C. Newell, Propagation of nonlinear wave envelopes, J. Math. Phys. 46, 133 (1967).

[18] V. E. Zakharov, Stability of periodic waves of finite amplitude on a surface of a deep fluid, J. Appl. Mech. Tech. Phys. 9, 190 (1968).
[19] G. Dattoli, F. P. Orisitto, and A. Torre, Evidence for multistability of light solitons in $\mathrm{SF}_{6}$ absorption measurements, Opt. Lett. 14, 456 (1989).

[20] A. E. Kaplan, Bistable solitons, Phys. Rev. Lett. 55, 1291 (1985).

[21] R. H. Enns, S. S. Rangnekar, and A. E. Kaplan, 'Robust' bistable solitons of the highly nonlinear Schrödinger equation, Phys. Rev. A 36, 1270 (1987).

[22] R. H. Enns and D. E. Edmundson, Guide to fabricating bistable-soliton-supporting media, Phys. Rev. A 47, 4524 (1993).

[23] A. Kumar, T. Kurz, and W. Lauterborn, Bistable solitons in triply doped fibers, Phys. Lett. A 235, 367 (1997).

[24] D. I. Pushkarov and S. Tanev, Bright and dark solitary wave propagation and bistability in the anomalous dispersion region of optical waveguides with third- and fifth-order nonlinearities, Opt. Commun. 124, 354 (1996).

[25] S. Tanev and D. I. Pushkarov, Solitary wave propagation and bistability in the normal dispersion region of highly nonlinear optical fibres and waveguides, Opt. Commun. 141, 322 (1997).

[26] A. Kumar and A. Kumar, Properties of bright solitons in averaged and unaveraged models for SGD fibres, Opt. Commun. 125, 377 (1996).

[27] C. Zhou, X. T. He, and S. Chen, Basic dynamic properties of the high-order nonlinear Schrödinger equation, Phys. Rev. A 46, 2277 (1992).

[28] D. Artigas, L. Torner, J. P. Torres, and N. Akhmediev, Asymmetrical splitting of higher-order optical solitons induced by quintic nonlinearity, Opt. Commun. 143, 322 (1997).

[29] P. Honzatko, Interaction of two quasi-solitons in semiconductordoped optical fibres, Opt. Commun. 127, 363 (1996).

[30] A. Chowdury, D. J. Kedziora, A. Ankiewicz, and N. Akhmediev, Soliton solutions of an integrable nonlinear Schrödinger equation with quintic terms, Phys. Rev. E 90, 032922 (2014).

[31] R. Sahadevan and L. Nalinidevi, Integrability of certain deformed nonlinear partial differential equations, J. Nonl. Math. Phys. 17, 379 (2010).

[32] R. Hirota, Exact envelope-soliton solutions of a nonlinear wave equation, J. Math. Phys. 14, 805 (1973).

[33] A. Ankiewicz, J. M. Soto-Crespo, and N. Akhmediev, Rogue waves and rational solutions of the hirota equation, Phys. Rev. E 81, 046602 (2010).

[34] M. Lakshmanan, K. Porsezian, and M. Daniel, Effect of discreteness on the continuum limit of the Heisenberg spin chain, Phys. Lett. A 133, 483 (1988).

[35] K. Porsezian, M. Daniel, and M. Lakshmanan, On the integrability aspects of the one-dimensional classical continuum isotropic biquadratic Heisenberg spin chain, J. Math. Phys. 33, 1807 (1992). 
[36] K. Porsezian, Completely integrable nonlinear Schrödinger type equations on moving space curves, Phys. Rev. E 55, 3785 (1997).

[37] A. Ankiewicz and N. Akhmediev, Higher-order integrable evolution equation and its soliton solutions, Phys. Lett. A 378, 358 (2014).

[38] A. Ankiewicz, Yan Wang, S. Wabnitz, and N. Akhmediev, Extended nonlinear Schrödinger equation with higher-order odd and even terms and its rogue wave solutions, Phys. Rev. E 89, 012907 (2014).

[39] T. Kano, Normal form of nonlinear Schrödinger equation, J. Phys. Soc. Jpn. 58, 4322 (1989).

[40] S. M. Hoseini and T. R. Marchant, Solitary wave interaction and evolution for a higher-order Hirota equation, Wave Motion 44, 92 (2006).

[41] P. Wang, B. Tian, W. J. Liu, Q. X. Qu, M. Li, and K. Sun, Lax pair, conservation laws and n-soliton solutions for the extended Korteweg-de Vries equations in fluids, Eur. Phys. J. D 61, 701 (2011).

[42] G. P. Agrawal, Nonlinear fiber optics: its history and recent progress, J. Opt. Soc. Am. B 28, A1 (2011).

[43] S. Backus, Ch. G. Durfee III, G. Mourou, H. C. Kapteyn, and M. M. Murnane, 0.2-TW laser system at $1 \mathrm{kHz}$, Opt. Lett. 22, 1256 (1997).

[44] M. Tajiri and Y. Watanabe, Breather solutions to the focusing nonlinear Schrödinger equation, Phys. Rev. E 57, 3510 (1998).
[45] Q-Han Park and H. J. Shin, Parametric control of soliton light traffic by cw traffic light, Phys. Rev. Lett. 82, 4432 (1999).

[46] L. Li, Z. Li, S. Li, and G. Zhou, Modulation instability and solitons on a cw background in inhomogeneous optical fiber media, Opt. Commun. 234, 169 (2004).

[47] V. E. Zakharov and A. Gelash, Nonlinear stage of modulation instability, Phys. Rev. Lett. 111, 054101 (2013).

[48] N. Akhmediev, V. M. Eleonskii, and N. E. Kulagin, Generation of periodic trains of picosecond pulses in an optical fiber: exact solutions, Sov. Phys. JETP 62, 894 (1985).

[49] N. N. Akhmediev and V. I. Korneev, Modulation instability and periodic solutions of nonlinear Schrödinger equation, Theor. Math. Phys. 69, 1089 (1986).

[50] N. N. Akhmediev, V. I. Korneev, and N. V. Mitskevich, Nmodulation signals in a single-mode optical waveguide under nonlinear conditions, Zh. Eksp. Teor. Fiz. 94, 159 (1988) [Sov. Phys. JETP 67, 89 (1988)].

[51] N. N. Akhmediev, V. M. Eleonskii, and N. E. Kulagin, Exact first-order solutions of the nonlinear Schrödinger equation, Theor. Math. Phys. 72, 809 (1987).

[52] D. J. Kedziora, A. Ankiewicz, and N. N. Akhmediev, Secondorder nonlinear Schrödinger equation breather solutions in the degenerate and rogue wave limits, Phys. Rev. E 85, 066601 (2012). 